\title{
Analysis of $X(4140)$ like states and their radial excitations in QCD
}

\section{A. Turkan*}

Ozyegin University, Department of Natural and Mathematical Sciences, 34794 Istanbul Turkey.

E-mail: arzu.turkan@ozyegin.edu.tr

\section{H. Dag}

Ozyegin University, Department of Natural and Mathematical Sciences, 34794 Istanbul Turkey.

E-mail: huseyin.dag@ozyegin.edu.tr

In this work, we investigated the $X(4140)$ and like states and their radial excitations by using molecular and diquark-antidiquark currents which couple to scalar, axial vector and tensor states via $\mathrm{QCD}$ sum rules. In operator product expansion, we considered quark, gluon and mixed vacuum condansates up to dimension eight. For the ground states coupling to these currents, we found that masses are almost degenerate with $X(4140)$. For the excited states, we found that scalar and tensor currents are coupling to $D_{s}^{*} \overline{D_{s}^{*}}$ threshold. However for the axial vector currents, the mass of the first excited state is compatible with $X(4274)$. Thus we conclude that, $X(4274)$ might be the first radial excitation of $X(4140)$.

XVII International Conference on Hadron Spectroscopy and Structure - Hadron2017

25-29 September, 2017

University of Salamanca, Salamanca, Spain

*Speaker. 
The spectroscopy of hadrons have its fruitful period due to discoveries of many new particles which are non conventional $c \bar{c}$ states in B-factories. Among these states, $X(4140)$ was first observed by CDF in the decay $B^{+} \rightarrow X(4140) K^{+} \rightarrow J / \psi \phi K^{+}$[1]. It is later confirmed by CDF, CMS and D0 [2, 3, 4, 5]. Also CDF [2] and CMS [3] observed a second peak which is accompanying $X(4140)$ in the same experiments. At last, LHCb announced that both $X(4140)$ and $X(4274)$ have quantum numbers $J^{P C}=1^{++}[6]$. In theory, there are several studies investigating the internal structure of $X(4140)$ and $X(4274)$, by using different quantum numbers in the tetraquark scheme $[7,8,9,10,11,12,13,14,15,16,17,18,19,20]$. Among these studies, most of them predicted the masses consistent with experimantal results, however only two of them successfully estimated both the mass and the quantum numbers of $X(4140)$ consistent with experiments[7, 20]. For the $X(4274)$, there are only two studies $[20,21]$ and their results are good agreement with the LHCb. In which authors threated $X(4274)$ as an axial vector ground state.

The mass and the decay constant are the primary properties of a hadron, which are both measurable and computable. So the investigation of these properties is important to understand their internal structure, non perturbative picture and dynamics of the excited states. Therefore we were motivated by above mentioned reasons to compute the properties of the ground and first excited states of $X(4140)$ and like states, which were estimated in a previous work [22]. For this aim we used the two point QCD sum rule (QCDSR) methods and we considered both meson molecule and diquark-antidiquark currents with quantum numbers $J^{P C}=0^{++}, 1^{++}, 2^{++}$. In this work, $X$ and $X^{\prime}$ denote the ground and the first excited states coupling to selected currents.

We started with two point correlation function to obtain the sum rules for the masses and the decay constants of $X$ and $X^{\prime}$ which can be written as

$$
\Pi(q)=i \int d^{4} x e^{i q \cdot x}\left\langle 0\left|\mathscr{T}\left(J(x) J^{\dagger}(0)\right)\right| 0\right\rangle,
$$

where $J(x)$ is the interpolating current coupling to $X$ and $X^{\prime}$ system. We chose the following molecular currents

$$
\begin{gathered}
J^{(1)}(x)=\bar{s}^{i}(x) \gamma_{\mu} c^{i}(x) \bar{c}^{j}(x) \gamma^{\mu} s^{j}(x), \\
J_{\mu}^{(1)}(x)=\frac{1}{\sqrt{2}}\left(\bar{s}^{i}(x) \gamma_{5} c^{i}(x) \bar{c}^{j}(x) \gamma_{\mu} s^{j}(x)-\bar{s}^{i}(x) \gamma_{\mu} c^{i}(x) \bar{c}^{j}(x) \gamma_{5} s^{j}(x)\right), \\
J_{\mu \nu}^{(1)}(x)=\frac{1}{\sqrt{2}}\left(\bar{s}^{i}(x) \gamma_{\mu} c^{i}(x) \bar{c}^{j}(x) \gamma_{\nu} s^{j}(x)+\bar{s}^{i}(x) \gamma_{\nu} c^{i}(x) \bar{c}^{j}(x) \gamma_{\mu} s^{j}(x)\right),
\end{gathered}
$$

and the following diquark-antidiquark currents

$$
\begin{gathered}
J^{(2)}(x)=\varepsilon \widetilde{\varepsilon}\left(s^{j}(x) C \gamma_{\mu} c^{k}(x) \bar{s}^{m}(x) \gamma^{\mu} C \bar{c}^{n}(x)\right), \\
J_{\mu}^{(2)}(x)=\frac{\varepsilon \widetilde{\varepsilon}}{\sqrt{2}}\left(s^{j}(x) C \gamma_{5} c^{k}(x) \bar{s}^{m}(x) \gamma_{\mu} C \bar{c}^{n}(x)-s^{j}(x) C \gamma_{\mu} c^{k}(x) \bar{s}^{m}(x) \gamma_{5} C \bar{c}^{n}(x)\right), \\
J_{\mu \nu}^{(2)}(x)=\frac{\varepsilon \widetilde{\varepsilon}}{\sqrt{2}}\left(s^{j}(x) C \gamma_{\mu} c^{k}(x) \bar{s}^{m}(x) \gamma_{\nu} C \bar{c}^{n}(x)+s^{j}(x) C \gamma_{\nu} c^{k}(x) \bar{s}^{m}(x) \gamma_{\mu} C \bar{c}^{n}(x)\right)
\end{gathered}
$$


where $i, j, \ldots$ are color indices, the superscripts (1) and (2) denote the current has either molecular or diquark-antidiquark state, $\varepsilon=\varepsilon^{i j k}, \widetilde{\varepsilon}=\varepsilon^{i m n}, C$ is the charge conjugation matrix.

In QCDSR, the correlation function in Eq. 1 is evaluated in two ways. In the operator product expansion (OPE) part, the correlator is calculated at the level of quark and gluon fields and in the physical part, it is computed in terms of physical parameters of $X$ and $X^{\prime}$ states. By equating the coefficients of the same Lorentz structures appearing in these two representations, the QCDSR for the physical quantities are obtained.

In order to obtain the physical side, we inserted a complete set which has the same quantum numbers with the $X$ and $X^{\prime}$ states between the interpolating currents and performed integration over $x$, and obtained

$$
\Pi^{\text {Phys }}(q)=\frac{\langle 0|J| X(q)\rangle\left\langle X(q)\left|J^{\dagger}\right| 0\right\rangle}{m_{X}^{2}-q^{2}}+\frac{\left\langle 0|J| X^{\prime}(q)\right\rangle\left\langle X^{\prime}(q)\left|J^{\dagger}\right| 0\right\rangle}{m_{X^{\prime}}^{2}-q^{2}}+\ldots,
$$

where $m_{X}\left(m_{X^{\prime}}\right)$ is the mass of the ground state (first radially excited state), and dots denote the contributions coming from the higher states and continuum. The ground and first radial excited states have same quantum numbers, thus their matrix elements are defined in smilar manner. So we defined scalar, axial vector and tensor matrix elements as follows

$$
\begin{gathered}
\left\langle 0|J| X^{(\prime)}(q)\right\rangle=\lambda_{X^{(\prime)}}, \\
\left\langle 0\left|J_{\mu}\right| X^{(\prime)}(q)\right\rangle=\lambda_{X^{(\prime)}} \varepsilon_{\mu}^{(\prime)}, \\
\left\langle 0\left|J_{\mu v}\right| X^{(\prime)}(q)\right\rangle=\lambda_{X^{(\prime)}} \varepsilon_{\mu \nu}^{(\prime)},
\end{gathered}
$$

where $\varepsilon_{\mu}^{(\prime)}$ and $\varepsilon_{\mu \nu}^{(\prime)}$ are vector and tensor polarizations satisfying the necessary conditions. Then using vector and tensor summations, the correlation functions in the physical side were written in terms of Lorentz structures as

$$
\begin{gathered}
\Pi^{\text {Phys }}(q)=\frac{\lambda_{X}}{m_{X}^{2}-q^{2}}+\frac{\lambda_{X^{\prime}}}{m_{X^{\prime}}^{2}-q^{2}}, \\
\Pi_{\mu \nu}^{\text {Phys }}(q)=\left(\frac{\lambda_{X}}{m_{X}^{2}-q^{2}}+\frac{\lambda_{X^{\prime}}}{m_{X^{\prime}}^{2}-q^{2}}\right) g_{\mu v}+\text { other structures, } \\
\Pi_{\mu v \alpha \beta}^{\text {Phys }}(q)=\left(\frac{\lambda_{X}}{m_{X}^{2}-q^{2}}+\frac{\lambda_{X^{\prime}}}{m_{X^{\prime}}^{2}-q^{2}}\right)\left\{\frac{1}{2}\left(g_{\mu \alpha} g_{v \beta}+g_{\mu \beta} g_{v \alpha}\right)\right\} \\
+ \text { other structures. }
\end{gathered}
$$

In Eqns. (11 - 12), we explicitly showed the coefficients of the Lorentz structures which were selected in this work.

In the OPE side we followed the calculations of traditional QCDSR as were done in Refs. $[22,23]$ and we do not present its details. However we modified the continuum threshold to $s_{0}^{\prime}$ instead of the $s_{0}$, in order to isolate the ground state. After these modifications, equating the same 
Lorentz structures in both $\Pi^{\text {Phys }}$ and $\Pi^{\mathrm{OPE}}$ and applying Borel transformation, we obtained the sum rules for the excited $X^{\prime}$ states as

$$
\lambda_{X^{\prime}}^{2} e^{-m_{X^{\prime}}^{2} / M^{2}}=\int_{\Lambda}^{s_{0}^{\prime}} d s \rho^{\mathrm{OPE}}(s) e^{-s / M^{2}}-\lambda_{X}^{2} e^{-m_{X}^{2} / M^{2}},
$$

where $\Lambda=\left(2 m_{s}+2 m_{c}\right)^{2}, m_{X}$ and $\lambda_{X}$ are the mass and the decay constant of $X$. These parameters were previously calculated in Ref. [22] and we presented their values in Table 1. In Eq. (13), $\rho^{\mathrm{OPE}}(s)=\operatorname{Im}\left[\Pi^{O P E} / \pi\right]$ is the spectral density, and their expressions are the same as those presented in Ref. [22]. In order to estimate the mass of the $X^{\prime}$, we took the derivative of Eq. (13) with respect to $-1 / M^{2}$, and divided it to Eq. (13), and obtained

$$
m_{X^{\prime}}^{2}=\frac{\int_{\Lambda}^{s_{0}^{\prime}} d s s \rho^{\mathrm{OPE}}(s) e^{-s / M^{2}}-m_{X}^{2} \lambda_{X}^{2} e^{-m_{X}^{2} / M^{2}}}{\int_{\Lambda}^{s_{0}^{\prime}} d s \rho^{\mathrm{OPE}}(s) e^{-s / M^{2}}-\lambda_{X}^{2} e^{-m_{X}^{2} / M^{2}}} .
$$

Table 1: Values of the masses and decay constants of $X(4140)$ [22].

\begin{tabular}{ccl}
\hline Current & $\begin{array}{c}m_{X} \\
(\mathrm{GeV})\end{array}$ & $\begin{array}{c}\lambda_{X} \\
\left(\times 10^{-2} \mathrm{GeV}^{5}\right)\end{array}$ \\
\hline$J^{(1)}\left(0^{++}\right)$ & $4.15 \pm 0.14$ & $3.89 \pm 0.95$ \\
$J_{\mu}^{(1)}\left(1^{++}\right)$ & $4.15 \pm 0.13$ & $2.22 \pm 0.50$ \\
$J_{\mu \nu}^{(1)}\left(2^{++}\right)$ & $4.15 \pm 0.13$ & $4.20 \pm 0.95$ \\
\hline$J^{(2)}\left(0^{++}\right)$ & $4.14 \pm 0.14$ & $4.51 \pm 1.10$ \\
$J_{\mu}^{(2)}\left(1^{++}\right)$ & $4.14 \pm 0.13$ & $2.56 \pm 0.58$ \\
$J_{\mu \nu}^{(2)}\left(2^{++}\right)$ & $4.14 \pm 0.13$ & $4.76 \pm 1.09$ \\
\hline
\end{tabular}

The obtained sum rules include numerous parameters, i.e. masses of $\mathrm{c}$ and $\mathrm{s}$ quarks and quark, gluon and mixed condansates. In order to perform numerical analysis, we used the values of input parameters given in Table 2.

Table 2: Input parameters

\begin{tabular}{lc}
\hline Parameters & Values \\
\hline$m_{c}$ & $(1.275 \pm 0.025) \mathrm{GeV}[24]$ \\
$m_{s}$ & $(95 \pm 5) \mathrm{MeV}[24]$ \\
$\langle\bar{q} q\rangle$ & $(-0.24 \pm 0.01)^{3} \mathrm{GeV}^{3}[11]$ \\
$\langle\bar{s} s\rangle$ & $0.8\langle\bar{q} q\rangle[11]$ \\
$\left\langle\frac{\alpha_{s} G^{2}}{\pi}\right\rangle$ & $(0.012 \pm 0.004) \mathrm{GeV}^{4}[11]$ \\
$m_{0}^{2}$ & $(0.8 \pm 0.1) \mathrm{GeV}^{2}[11]$ \\
\hline
\end{tabular}

In addition to the input parameters, the derived sum rules also depend on $M^{2}$ and $s_{0}^{\prime}$. We determined the working regions of these parameters respecting the following criteria: sum rules 
should satisfy the convergence of OPE and dominance of the pole contribution. For each current, we fixed the working regions of the $M^{2}$ in which the sum rules satisfy both pole dominance and OPE convergence, and chose the upper bound of the $s_{0}^{\prime}$ as $22.6 \mathrm{GeV}^{2}$ to isolate the contributions of "ground + first excited states" from the continuum. For the lower bound of $s_{0}^{\prime}$, we applied a linear fit and expressed the minimum value of $s_{0}^{\prime}$ in terms of $M^{2}$. We present the working regions of $M^{2}$, $s_{0}^{\prime}$ and the values of the masses of $X^{\prime}$ states in Table 3.

Table 3: Working regions of parameters $M^{2}, s_{0}^{\prime}$, and the values of the masses of $X^{\prime}$ states.

\begin{tabular}{lcccc}
\hline Current & $\begin{array}{c}M_{\min }^{2} \\
\left(\mathrm{GeV}^{2}\right)\end{array}$ & $\begin{array}{c}M_{\max }^{2} \\
\left(\mathrm{GeV}^{2}\right)\end{array}$ & $\begin{array}{c}22.6 \geq s_{0} \geq \\
\left(\mathrm{GeV}^{2}\right)\end{array}$ & $m_{X^{\prime}}(\mathrm{GeV})$ \\
\hline$J^{(1)}\left(0^{++}\right)$ & 4.0 & 4.5 & $3.75 M^{2}+5.88$ & $4.22 \pm 0.11$ \\
$J_{\mu}^{(1)}\left(1^{++}\right)$ & 4.3 & 4.8 & $3.66 M^{2}+5.10$ & $4.48 \pm 0.41$ \\
$J_{\mu \nu}^{(1)}\left(2^{++}\right)$ & 4.3 & 4.8 & $3.53 M^{2}+5.76$ & $4.21 \pm 0.11$ \\
\hline$J^{(2)}\left(0^{++}\right)$ & 3.9 & 4.4 & $3.85 M^{2}+5.65$ & $4.22 \pm 0.12$ \\
$J_{\mu}^{(2)}\left(1^{++}\right)$ & 4.2 & 4.7 & $3.75 M^{2}+5.13$ & $4.30 \pm 0.23$ \\
$J_{\mu \nu}^{(2)}\left(2^{++}\right)$ & 4.1 & 4.6 & $3.33 M^{2}+7.27$ & $4.23 \pm 0.10$ \\
\hline
\end{tabular}

After the numerical analysis, we found that the masses estimated by scalar and tensor currents coupling to first radial excitations are almost the same as the $D_{s}^{*} \bar{D}_{s}^{*}$ threshold. These excited states might be very loosely bound $D_{s}^{*} \bar{D}_{s}^{*}$ resonances. This might also be a sign of dominant molecular content for ground states of the scalar and tensor partners of axial vector $X(4140)$. On the other hand, we found that the mass of the first radial excitation of axial vector $X(4140)$ is compatible with $X(4274)$.

In summary, we performed a QCD sum rules analysis for possible $D_{s}^{*} \bar{D}_{s}^{*}$ molecule and diquarkantidiquark currents with $J^{P C}=0^{++}, 1^{++}$and $2^{++}$, and estimated the masses of the first radial excitations of the ground states coupling to these currents. To extract masses of the excited states from the sum rules, we modified the continuum threshold and separated "ground + first excited state" from the continuum. As a result, we concluded that $X(4274)$ can be identified as the first radial excitation of axial vector $X(4140)$ with diquark-antidiquark content.

\section{ACKNOWLEDGEMENTS}

This work is supported by TUBITAK under project number 114F215. H. D. and A. T. thank to K. Azizi, H. Sundu and A. Ozpineci for helpful discussions. A. Turkan also thanks to organizers of HADRON17 for organizing a fruitful conference.

\section{References}

[1] T. Aaltonen et al. [CDF Collaboration], Phys. Rev. Lett. 102, 242002 (2009).

[2] T. Aaltonen et al. [CDF Collaboration], arXiv:1101.6058v1 [hep-ex], (2011). 
[3] S. Chatrchyan et al. [CMS Collaboration], Phys. Lett. B 734, 261 (2014), arXiv:1309.6920v1 [hep-ex].

[4] V. M. Abazov et al. (D0 Collaboration), Phys. Rev. D 89, 012004 (2014).

[5] V. M. Abazov et al. (D0 Collaboration), Phys. Rev. Lett. 115, 232001 (2015).

[6] R. Aaij et al. [LHCb Collaboration], Phys. Rev. Lett. 118, 022003 (2017); R. Aaij et al. [LHCb Collaboration], Phys. Rev. D 95, 012002 (2017).

[7] F. Stancu, J. Phys. G 37, 075017 (2010), arXiv:0906.2485 [hep-ph].

[8] N. Mahajan, Phys. Lett. B 679228 (2009), arXiv:0903.3107 [hep-ph].

[9] T. Branz, T. Gutsche, V. E. Lyubovitskij, AIPConf. Proc.1257 432 (2010), arXiv:1001.3959v1 [hep-ph].

[10] M. Padmanath, C. B. Lang, S. Prelovsek, Phys. Rev. D 92034501 (2015), arXiv:1503.03257 [hep-lat].

[11] Raphael M. Albuquerque, Mirian E. Bracco, Marina Nielsen, Phys.Lett. B 678 (2009) 186-190, arXiv:0903.5540 [hep-ph].

[12] Jian-Rong Zhang and Ming-Qiu Huang, J. Phys. G 37, 025005,(2010), arXiv:0905.4178 [hep-ph].

[13] Jian-Rong Zhang and Ming-Qiu Huang, Commun. Theor. Phys. 54, 1075-1090 (2010), arXiv:0905.4672 [hep-ph].

[14] Zhi-Gang Wang, Eur. Phys. J. C 74, 2963,(2014), arXiv:1403.0810 [hep-ph].

[15] Zhi-Gang Wang, Zhi-Cheng Liu, Xiao-Hong Zhang, Eur. Phys. J. C 64, 373-386,(2009), arXiv:0907.1467 [hep-ph].

[16] Zhi-Gang Wang, Eur. Phys. J. C 63, 115-122, (2009), arXiv:0903.5200 [hep-ph].

[17] Zhi-Gang Wang and Ye-Fan Tian, Int. J. Mod. Phys. A 30, 1550004 (2015), arXiv:1502.04619 [hep-ph].

[18] Zhi-Gang Wang, Eur. Phys. J. C 76 (2016) 657, arXiv:1607.00701 [hep-ph].

[19] W. Chen and S. L. Zhu, Phys. Rev. D 83, 034010 (2011), arXiv:1010.3397 [hep-ph].

[20] S. S. Agaev, K. Azizi, H. Sundu, Phys. Rev. D 95114003 (2017), arXiv:1703.10323 [hep-ph].

[21] Hua-Xing Chen, Er-Liang Cui, Wei Chen, Xiang Liu,Shi-Lin Zhu, Eur. Phys. J. C 77160 (2017), arXiv:1606.03179v3 [hep- ph].

[22] H. Dag and A. Turkan, arXiv:1705.02587[hep-ph].

[23] H. Dag and A. Turkan,âĂIIInvestigating the Structure of X(4140) in QCD âĂİ, EPJ Web of Conf., 137 06007 (2017), Proceedings, XIIth Quark Confinement and the Hadron Spectrum, (2016).

[24] C. Patrignani et al. (Particle Data Group), Chin. Phys. C 40, 100001 (2016). 